\title{
Polysaccharide hemostatic powder to prevent bleeding after endoscopic submucosal dissection in high risk patients: a randomized controlled trial $\square$
}

Authors

Da Hyun Jung ${ }^{*}, 1$, Hee Seok Moon ${ }^{*}, 2 \oplus$, Chan Hyuk Park ${ }^{3} \odot$, Jun Chul Park ${ }^{\oplus}$

Institutions

1 Department of Gastroenterology, Internal Medicine, Severance Hospital, Yonsei University College of Medicine, Seoul, Republic of Korea

2 Department of Internal Medicine, Chungnam National University Hospital, Chungnam National University College of Medicine, Daejeon, Republic of Korea

3 Department of Internal Medicine, Hanyang University Guri Hospital, Hanyang University College of Medicine, Seoul, Republic of Korea

submitted 9.6.2020

accepted after revision 17.11.2020

published online 17.11.2020

Bibliography

Endoscopy 2021; 53: 994-1002

DOI 10.1055/a-1312-9420

ISSN 0013-726X

(C) 2020. Thieme. All rights reserved.

Georg Thieme Verlag KG, Rüdigerstraße 14,

70469 Stuttgart, Germany

\# Fig. 1s, 2s, Table 1s

Supplementary material is available under

https://doi.org/10.1055/a-1312-9420

Scan this QR-Code for the author commentary.

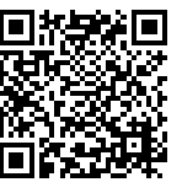

Corresponding author

Jun Chul Park, MD, PhD, Division of Gastroenterology, Department of Internal Medicine, Yonsei University College of

Medicine, 50-1 Yonsei-ro, Seodaemun-gu, Seoul 03722,

Republic of Korea

JUNCHUL75@yuhs.ac

\section{ABSTRACT}

Background Bleeding after endoscopic submucosal dissection (ESD) is a severe adverse event. Several methods to prevent post-ESD bleeding (PEB) have been introduced; however, they have not been widely used because of technical difficulties. We aimed to investigate whether polysaccharide hemostatic powder (PHP), which is very easy to apply, can prevent early post-ESD bleeding, especially in patients with a high risk of post-ESD bleeding.

Methods This was a prospective, multicenter, randomized, open-label, controlled trial. Patients with a high risk for post-ESD bleeding were enrolled. Patients with gastric neoplasms in whom the resected specimen size was expected to be $>40 \mathrm{~mm}$ and those who were regularly taking antithrombotic agents were defined as high risk patients. Patients were randomly assigned to the PHP or control groups.

Results Between May 2017 and September 2018, 143 patients were enrolled (PHP group, 73; control group, 70). The total post-ESD bleeding rate was $6.3 \%$ (PHP group, $5.5 \%$ vs. control group, $7.1 \% ; P=0.74$ ). There was no bleeding within 7 days after ESD in the PHP group. Continued antithrombotic use was an independent risk factor for post-ESD bleeding. In subgroup analysis excluding the patients who continued to take antithrombotic agents $(n=129)$ during ESD, the rate of post-ESD bleeding tended to be lower in the PHP group than in the control group ( $0 \%$ vs. $6.3 \% ; P=0.06$ ).

Conclusion PHP did not demonstrate a significant effect on the prevention of post-ESD bleeding in this study. Further larger scale, randomized controlled trials are needed to confirm this. 


\section{Introduction}

Endoscopic submucosal dissection (ESD) has been recommended as a standard treatment for gastric neoplasms and can provide excellent long-term survival [1,2]. Most ESD procedures are safe and feasible; however, adverse events such as bleeding, perforation, and pneumonia may occur after the procedure [3]. Post-ESD bleeding is one of the most common adverse events after ESD; its incidence has been estimated to be about $5 \%$ [48]. However, the incidence of bleeding, especially in high risk patients, could range from $15 \%$ to $57.3 \%$ [9-11].

Previous studies have demonstrated that a resected specimen size $>40 \mathrm{~mm}$, lesions located in the upper third of the stomach, and the use of anticoagulants or antiplatelet agents are significant risk factors for post-ESD bleeding [5, 12-14]. With an aging society, many elderly patients have been prescribed antithrombotic agents because of the increasing incidence of cerebrovascular accidents and cardiovascular disease [10]. Moreover, patients with large lesions, part of the expanded indications for ESD, are also increasing [15]. Consequently, many high risk patients now undergo ESD. In addition, bleeding after ESD could be fatal in these patients.

Recently, several methods for preventing post-ESD bleeding have been introduced [14]. Closing methods, such as clipping with a detachable snare and endoscopic suturing, have been shown to promote earlier healing of ulcers and reduce the need for hospitalization after ESD $[16,17]$. One recent study reported that the application of polyglycolic acid sheets and fibrin glue showed promising efficacy in the prevention of postESD bleeding in high risk patients [18]. However, these methods are not easy to use because they require technical skills equivalent to those needed for ESD.

Polysaccharide hemostatic powder (PHP) is a novel hemostatic, absorbable, modified polymer particle powder. It is being used clinically as it is easy to apply, regardless of the anatomical location. Our previous preliminary study [19] demonstrated the possibility that PHP has efficacy in preventing early post-ESD bleeding in high risk patients (those with a large resection owing to a specimen size $\geq 40 \mathrm{~mm}$ or those taking antithrombotic agents). However, no prospective randomized controlled trials (RCTs) have been conducted. Therefore, we designed the first RCT aiming to determine the efficacy of PHP in preventing post-ESD bleeding, especially in high risk patients.

\section{Methods}

\section{Patients}

Between May 2017 and September 2018, we enrolled patients who underwent ESD for gastric neoplasms and were at a high risk for post-ESD bleeding into this multicenter, open-label, prospective RCT conducted at four referral institutions across South Korea. High risk patients were defined as patients with gastric neoplasms whose resected specimen size was expected to be $>40 \mathrm{~mm}$ or who were regularly taking antithrombotic agents. Written informed consent was obtained from all patients before study enrollment.
The inclusion criteria were: (i) at least one of the high risk criteria mentioned above; (ii) age 20-85 years; (iii) Eastern Cooperative Oncology Group (ECOG) performance status 0 or 1; (iv) pathological diagnosis of gastric adenoma or cancer eligible for ESD; and ( $v$ ) absolute neutrophil count $\geq 1500 / \mathrm{mm}^{3}$, hemoglobin $\geq 9 \mathrm{~g} / \mathrm{dL}$, platelets $\geq 100,000 / \mathrm{mm}^{3}$, international normalized ratio $($ INR) $\leq 1.5$, activated partial thromboplastin time $\leq$ $1.5 \times$ normal value $(\mathrm{IU} / \mathrm{L})$, total bilirubin $<1.5 \times$ normal value $(\mathrm{mg} / \mathrm{dL})$, aspartate aminotransferase and alanine aminotransferase $\leq 3.0 \times$ normal value $(\mathrm{IU} / \mathrm{L})$, alkaline phosphatases $<3.0 \times$ normal value $(\mathrm{IU} / \mathrm{L})$, and creatinine $\leq 1.5 \mathrm{mg} / \mathrm{dL}$ or calculated creatinine clearance $\geq 60 \mathrm{~mL} / \mathrm{min}$ in laboratory blood tests performed within 1 month before enrollment.

The exclusion criteria were: (i) history of allergy to starch or starch-derived components; (ii) history of upper gastrointestinal (GI) surgery; (iii) local recurrence at the previous ESD site; (iv) significant cardiovascular, renal, hepatic, neurotic, or psychological disorders; and (v) pregnant or currently breastfeeding.

The management of antithrombotic agents was defined as described below. (i) Patients taking aspirin or clopidogrel monotherapy for a history of coronary artery occlusive disease or cerebrovascular disease were referred to a cardiologist or neurologist with regard to the feasibility of discontinuing aspirin during the perioperative period. If they had a low thromboembolism risk, aspirin was stopped 5 days before the procedure and reinitiated 3-5 days after the procedure. (ii) Patients taking both aspirin and clopidogrel because of a history of coronary artery occlusive disease or cerebrovascular disease were referred to a cardiologist or a neurologist about the feasibility of discontinuing antithrombotic therapy during the perioperative period. Only aspirin was continued, and clopidogrel was discontinued 5 days before the procedure and reinitiated 3-5 days after the procedure. (iii) Patients taking anticoagulants were also referred to a cardiologist or neurologist about the feasibility of discontinuing anticoagulant therapy during the perioperative period. Anticoagulants were discontinued 5 days before the procedure and reinitiated 24 hours after the procedure. (iv) Patients taking anticoagulants who needed heparin bridging therapy discontinued anticoagulants 5 days before the procedure and received low molecular weighted heparin $(\mathrm{LMWH})$ injections at $100 \mathrm{U} / \mathrm{kg}$ once a day from 3 days before the procedure. The patients were not injected with LMWH on the day before the procedure; they took an anticoagulant on the evening of the procedure and were injected with LMWH $100 \mathrm{U} / \mathrm{kg}$ once a day on the day of the procedure and the next day. The continuous use of antithrombotic agents was defined as the continuous use of antithrombotic agents or discontinuation less than 1 day pre-ESD.

A single study coordinator performed the randomizations using a computer-generated randomization table. Patients were randomly assigned to one of two groups (PHP or control). Randomization was made immediately after ESD including last hemostasis. Therefore, the endoscopist could not know which group would be assigned during the ESD procedure.

The study protocol was approved by the institutional review board of each participating institution. 

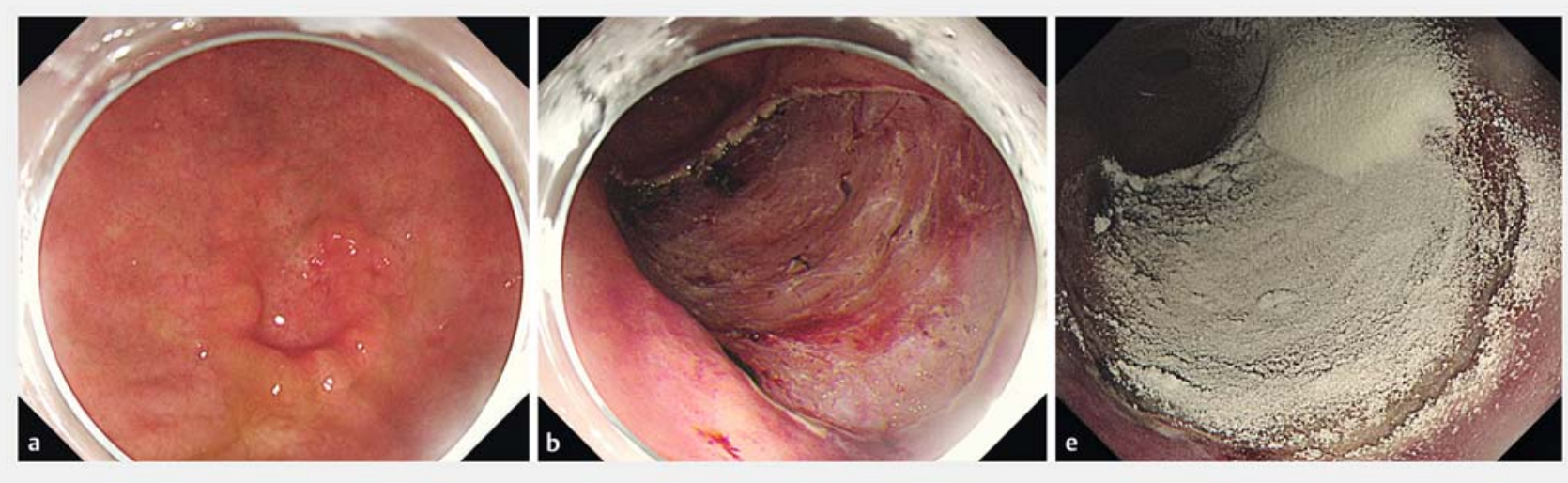

- Fig. 1 Endoscopic views showing: a a slightly depressed early gastric cancer at the antrum; b a post-resection ulcer (maximum diameter 58 $\mathrm{mm}$ ) after completion of endoscopic submucosal dissection; $\mathbf{c}$ the appearance after application of polysaccharide hemostatic powder to the post-resection ulcer surface.

\section{ESD techniques and PHP application}

ESD was performed according to the standard procedure sequence: (i) marking in the surrounding area of the lesion via argon plasma coagulation or DualKnife (Olympus, Tokyo, Japan); (ii) submucosal injection of a mixture of $0.8 \%$ indigo carmine, normal saline, and epinephrine $(0.01 \mathrm{mg} / \mathrm{mL})$; (iii) circumferential incision (precut) along the outer border of the lesion and dissection of the submucosal layer with an ESD device (needle knife, insulated-tip knife, and/or DualKnife [Olympus]); (iv) meticulous endoscopic hemostasis with a hemoclip or hemostatic forceps (FD-410LR; Olympus) for bleeding or exposed vessels.

Hemostasis was performed as needed during the procedure as well as for oozing or exposed vessels after ESD completion. In the control group, the hemostasis sequence was the last procedure. In the PHP group, after removal of the specimen and the final hemostasis sequence, $2 \mathrm{~g}$ PHP (Endo-Clot Plus, Inc., Santa Clara, California, USA) was applied as previously described ( Fig. 1; V Video 1) [19]. PHP was sprayed onto the surface of the post-ESD ulcer site using a catheter passed through the working channel of the endoscope under continuous air flow.

\section{Perioperative management and post-ESD follow-up}

All patients received an intravenous injection of $40 \mathrm{mg}$ pantoprazole twice daily for the first 2 days after ESD. Beginning on the third day after ESD, the patients took $30 \mathrm{mg}$ lansoprazole orally once a day for 28 days. All patients underwent chest and abdominal radiography and complete blood count analysis immediately after the procedure and on the next day after ESD, to check for adverse events such as pneumonia, bleeding, or perforation. They were discharged on the third day if there were no reported adverse events. All patients were instructed to visit the outpatient department at 2 and 4 weeks after ESD for the evaluation of post-procedural bleeding. Blood tests were performed at both 2 and 4 weeks after ESD.

Post-ESD bleeding was defined as clinical or laboratory signs of bleeding with urgent endoscopy findings showing bleeding in the stomach or a requirement for endoscopic hemostasis. Endoscopic hemostasis was performed in lesions classified as
Forrest la, Ib, or Ila. Clinical symptoms such as melena, hematemesis, or hematochezia were defined as bleeding signs, and the laboratory sign was defined as a decrease in hemoglobin level by $\geq 2.0 \mathrm{~g} / \mathrm{dL}$.

\section{Study outcomes}

The primary outcome, to investigate the efficacy of PHP for preventing post-ESD bleeding compared with that of conventional therapy, was the post-ESD bleeding rate up until 4 weeks after ESD. Moreover, the risk factors for post-ESD bleeding were analyzed. The secondary outcomes were the post-ESD bleeding rate in patients with a resected lesion of $>40 \mathrm{~mm}$ and adverse events associated with the use of PHP.

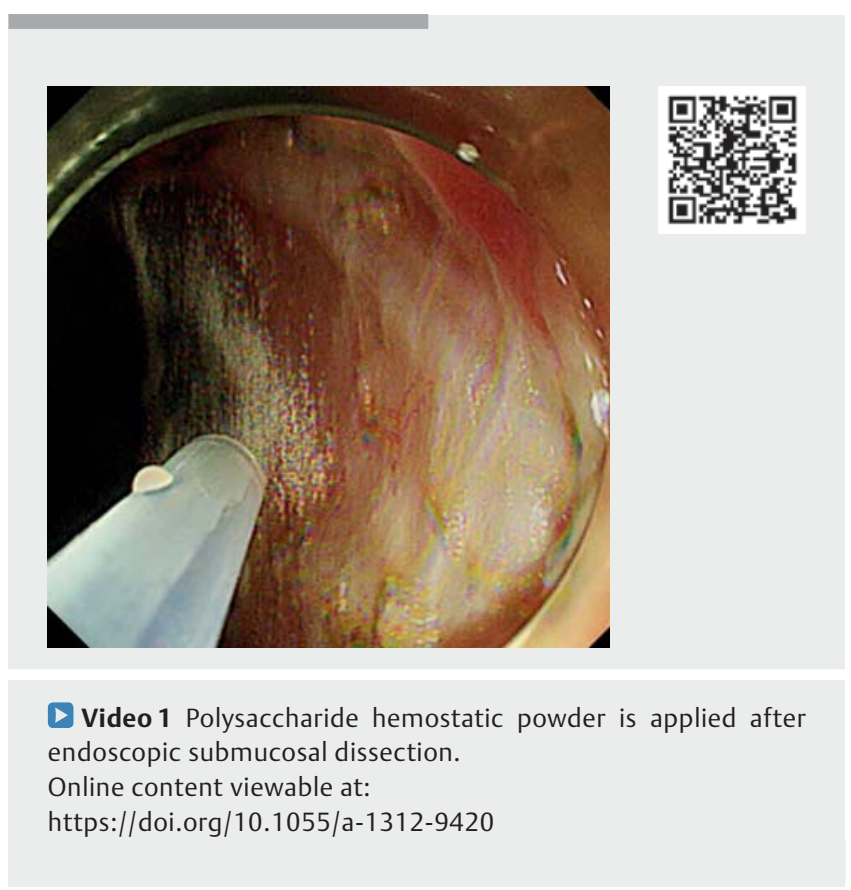




\section{Statistical analysis}

Continuous variables are presented as mean (standard deviation) and discrete variables as frequency (\%). The chi-squared test or Fisher's exact test were used to compare categorical parameters. Student's $t$ test was used to compare continuous variables. For the evaluation of the independent risk factors associated with bleeding, we used logistic regression analysis (univariate and multivariate regression). In addition, we verified the variance inflation factor (VIF) to check multicollinearity and confirmed that the VIF values of risk factors were 10 or less. Factors were considered significant at a two-sided $P$ value of $<0.05$.

According to our previously presented prediction model for post-ESD bleeding based on our prospective cohort database, the post-ESD bleeding rate in high risk patients was over $30 \%$ [20]. In addition, based on other published studies [8, 19], we estimated that the post-ESD bleeding rate would be $30 \%$ in the control group and $10 \%$ in the PHP group, particularly high risk patients. The sample size was calculated using a significance level of 0.05 and statistical power of 0.8 , based on a chisquared test. The calculated sample size was 62 patients in each arm, meaning a total of 143 patients to allow for a $15 \%$ dropout rate.

Statistical analyses were performed using SPSS version 23.0 for Windows (SPSS Inc., Chicago, Illinois, USA).

\section{Results}

\section{Baseline characteristics of patients and ESD outcomes}

We enrolled 143 eligible patients from May 2017 to September 2018 at four institutions across South Korea. Of these, 73 were assigned to the PHP group and 70 to the control group (Fig. 1 s, see online-only Supplementary material). Their baseline characteristics are shown in $>$ Table 1 . There was no difference in the baseline characteristics between the PHP and control groups. A total of 38 patients were taking antithrombotic agents. Among them, 34 patients were taking antithrombotic monotherapy and four patients were taking dual antithrombotic drugs. With regard to the management of antithrombotic therapy, 24 patients stopped their antithrombotic agents, whereas eight patients in the PHP group and six patients in the control group continued their antithrombotic agents.

The resected lesion size was $48.9 \mathrm{~mm}$ in the PHP group and $46.9 \mathrm{~mm}$ in the control group $(P=0.24)$. Moreover, the resection time and preventive coagulation time were not significantly different between the two groups. The en bloc resection rate of all ESD procedures was $99.3 \%$. Furthermore, the complete resection and curative resection rates were $93.7 \%$ and $90.9 \%$, respectively. There were no cases of intraoperative perforation and no other adverse events, except for post-ESD bleeding.

The tumor size was $18.4 \mathrm{~mm}$ in the PHP group and $19.3 \mathrm{~mm}$ in the control group $(P=0.66)$. In the PHP group, there were 21 adenomas (28.8\%) and 52 carcinomas (71.2\%). In the control group, there were 21 adenomas (30.0\%) and 49 carcinomas
(70.0\%). The procedure time to shield the artificial ulcers was less than 2 minutes.

\section{Primary outcome}

Post-ESD bleeding occurred in 5.5\% (4/73) and 7.1\% (5/70) of patients in the PHP and control groups, respectively ( $>\mathrm{Ta}$ ble 2 ). The post-ESD bleeding rate was not significantly different between the groups $(P=0.74)$. The details of the post-ESD bleeding patients are shown in Table $1 \mathrm{~s}$. The median (range) timing of bleeding after ESD was 13 (8-19) days in the PHP group and 12 (3-18) days in the control group. There was no bleeding within 7 days after ESD in the PHP group (Fig. 2s).

Endoscopic hemostasis was achieved in all cases of bleeding, and the proportion of high risk bleeding lesions (Forrest la, Ib, or Ila) requiring endoscopic hemostasis was lower in the PHP group $(1 / 4 ; 25 \%)$ than in the control group $(2 / 5 ; 40 \%)$. Other lesions that required endoscopic hemostasis were Forrest IIb (2/4) and III (1/4) lesions in the PHP group and Forrest IIb (1/5) and III (2/5) lesions in the control group. Complete hemostasis was successfully achieved for all high risk bleeding lesions.

\section{Secondary outcomes}

A total of 138 resected lesions were $>40 \mathrm{~mm}$. For these lesions, the post-ESD bleeding rates were $2.9 \%(2 / 69)$ and $7.2 \%(5 / 69)$ in the PHP and control groups, respectively $(P=0.44)(\triangleright$ Table 2$)$. In subgroup analysis excluding the patients who continued to take antithrombotic agents $(n=129)$, the rate of post-ESD bleeding showed a tendency toward being lower in the PHP group than in the control group ( $0 \%$ vs. $6.3 \% ; P=0.06$ ). There were no adverse events associated with the application of PHP.

\section{Risk factors for post-ESD bleeding}

In univariate analysis, co-morbidities such as diabetes mellitus $(P=0.01)$, coronary artery disease $(P=0.04)$, antithrombotic use $(P=0.01)$, multiple antithrombotic use $(P=0.02)$, and continued antithrombotic use $(P<0.001)$ were significant risk factors for post-ESD bleeding. In multivariate analysis, only continued antithrombotic use was an independent risk factor for post-ESD bleeding ( $>$ Table 3 ).

\section{Discussion}

This is the first RCT to investigate the efficacy of the application of PHP for the prevention of post-ESD bleeding. Previously, we have reported the efficacy of PHP in preventing bleeding after gastric ESD in high risk patients [19]. PHP application is a new hemostatic method for upper Gl bleeding that works topically [21]. One of advantages of hemostatic powder is that its use requires minimal technical expertise. It is an effective way of shielding the post-resection ulcer surface and accelerating the physiologic clotting system.

In our previous study, the application of PHP showed efficacy in preventing early post-ESD bleeding in high risk patients with a large resected lesion ( $>40 \mathrm{~mm}$ ) or on regular antithrombotic use. However, the present RCT could not demonstrate signifi- 
- Table 1 Baseline characteristics of the 143 patients enrolled in the study who underwent endoscopic submucosal dissection (ESD) for gastric neoplasms and were at high risk for post-ESD bleeding, along with details of their endoscopic procedures and pathologic diagnoses.

\begin{tabular}{|l|l|l|l|}
\hline Variables & PHP \\
$(n=73)$ & $\begin{array}{l}\text { Control } \\
(n=70)\end{array}$ & $P$ value \\
\hline
\end{tabular}

\section{Patients}

\begin{tabular}{|c|c|c|c|}
\hline Age, mean (SD), years & $65.4(8.8)$ & $65.3(9.7)$ & 0.95 \\
\hline Sex, n (\%) & & & $>0.99$ \\
\hline - Male & $54(74.0)$ & $51(72.9)$ & \\
\hline - Female & $19(26.0)$ & $19(27.1)$ & \\
\hline \multicolumn{4}{|l|}{ Co-morbidity, n (\%) } \\
\hline - Hypertension & $37(50.7)$ & $29(41.4)$ & 0.32 \\
\hline - Diabetes mellitus & $18(24.7)$ & $16(22.9)$ & 0.85 \\
\hline - Coronary artery disease & $8(11.0)$ & $5(7.1)$ & 0.56 \\
\hline - Cerebrovascular disease & $9(12.3)$ & $5(7.1)$ & 0.40 \\
\hline - Chronic kidney disease & $4(5.5)$ & $3(4.3)$ & $>0.99$ \\
\hline Antithrombotic agents use, $\mathrm{n}(\%)$ & $20(27.4)$ & $18(25.7)$ & 0.85 \\
\hline - Aspirin & $12(16.4)$ & $10(14.3)$ & 0.82 \\
\hline - Clopidogrel & $9(12.3)$ & $5(7.1)$ & 0.40 \\
\hline - Other antiplatelets & $2(2.7)$ & $3(4.3)$ & 0.68 \\
\hline - Anticoagulant use & $0(0.0)$ & $1(1.4)$ & 0.49 \\
\hline Multiple antithrombotic agent use, n (\%) & $3(4.1)$ & $1(1.4)$ & 0.62 \\
\hline \multicolumn{4}{|l|}{ Antithrombotic management, n (\%) } \\
\hline - Continued use & $8(11.0)$ & $6(8.6)$ & 0.78 \\
\hline \multicolumn{4}{|l|}{ ESD procedure } \\
\hline \multicolumn{3}{|l|}{ Tumor location, n (\%) } & \multirow[t]{4}{*}{0.53} \\
\hline - Lower third & $65(89.0)$ & $58(82.9)$ & \\
\hline - Middle third & $4(5.5)$ & $5(7.1)$ & \\
\hline - Upper third & $4(5.5)$ & $7(10.0)$ & \\
\hline Estimated size more than 40 mm, n (\%) & $69(94.5)$ & $69(98.6)$ & 0.37 \\
\hline Resected lesion size, mean (SD), mm & $48.9(11.4)$ & $46.9(9.2)$ & 0.24 \\
\hline Resection time, mean (SD), minutes & $26.2(23.4)$ & $30.5(33.7)$ & 0.37 \\
\hline Preventive coagulation time, mean (SD), minutes & $6.4(6.8)$ & $5.6(2.8)$ & 0.36 \\
\hline En bloc resection, $\mathrm{n}(\%)$ & $72(98.6)$ & $70(100.0)$ & $>0.99$ \\
\hline Complete resection, $\mathrm{n}(\%)$ & $70(95.9)$ & $64(92.8)$ & 0.49 \\
\hline Curative resection, n (\%) & $69(94.5)$ & $61(87.1)$ & 0.15 \\
\hline Intraprocedural perforation, n (\%) & $0(0.0)$ & $0(0.0)$ & - \\
\hline \multicolumn{4}{|l|}{ Pathologic diagnosis } \\
\hline Tumor size, mean (SD), mm & $18.4(12.2)$ & $19.3(10.3)$ & 0.66 \\
\hline \multicolumn{3}{|l|}{ Histology, n (\%) } & \multirow[t]{3}{*}{$>0.99$} \\
\hline - Adenoma & $21(28.8)$ & $21(30.0)$ & \\
\hline - Carcinoma & $52(71.2)$ & $49(70.0)$ & \\
\hline
\end{tabular}


- Table 2 Comparison of the rates of post-endoscopic submucosal dissection bleeding. All values are given as $\mathrm{n}(\%)$ [ $\mathrm{N}$ for that subgroup].

\begin{tabular}{|l|l|l|l|}
\hline & PHP & Control & P value \\
\hline Overall & $4(5.5)$ & $5(7.1)$ & 0.74 \\
{$[N=70]$} & $5(7.2)$ \\
\hline Patients with resection size $\geq 40 \mathrm{~mm}$ & {$[\mathrm{~N}=73]$} & {$[\mathrm{N}=69]$} \\
\hline Patients with cessation of antithrombotic agents & $2(2.9)$ & $4(6.3)$ \\
\hline Patients on antithrombotic agents, $\mathrm{N}$ & {$[\mathrm{N}=69]$} & {$[\mathrm{N}=64]$} \\
\hline & $0(0.0)$ & $2(11.1)$ \\
\hline PHP, polysaccharide hemostatic powder. & {$[\mathrm{N}=65]$} & {$[\mathrm{N}=18]$} \\
\hline
\end{tabular}

cantly greater efficacy of PHP in preventing post-ESD bleeding compared with that seen with conventional treatments.

One possible explanation is the post-ESD bleeding rate of high risk patients in this study, which is much lower than we expected in both groups. The reasons for the low post-ESD bleeding rate in the control group might be technical advancement of ESD and improved endoscopic capabilities since the previous period. According to our previously reported prediction model for post-ESD bleeding, based on our prospective cohort database, the rate among high risk patients was over $30 \%$. This cohort study included a total of 4143 patients who had undergone ESD from January 2007 to November 2016. However, the present RCT has been conducted very recently (from May 2017 and September 2018). Therefore, the technical improvements in ESD is one of most important reasons for the low post-ESD bleeding rate.

In addition, this RCT is an open-label study carried out in high risk patients particularly, so the endoscopists might have performed more fastidious hemostasis than usual. In general, advanced skills and adequate experience are required to reduce the risk of adverse events, including post-ESD bleeding [22]. According to a previous study [23], preventive coagulation of visible vessels in the resection area after ESD may lead to a lower bleeding rate. Because muscle injury during dissection is also a risk factor for post-ESD bleeding, these two factors are the most important risk factors for post-ESD bleeding. In addition, meticulous hemostasis of all visible vessels after ESD could decrease post-ESD bleeding. For these reasons, it could also be that the post-ESD bleeding rate is lower than we expected because the participating endoscopists in this multicenter study were experts in ESD.

Although bleeding occurred in only four patients in the PHP group, post-ESD bleeding developed $>8$ days after ESD and most of the bleeding events occurred at $>2$ weeks. The mechanism of PHP is that it absorbs water to form a gel matrix that covers the ulcer surface for 3-48 hours and accelerates the physiologic clotting system by enhancing the local concentration of coagulating factors $[21,24]$. In animal models, PHP was able to stop active bleeding caused by snaring in pigs that were administered antithrombotic agents [25]. Therefore, PHP was considered to act on bleeding in the early phase, within 2-3 days. For this reason, post-ESD bleeding in the PHP group might have occurred as late-phase bleeding. According to previous studies [26, 27], acute and delayed post-ESD bleeding are associated with different clinical characteristics. Antithrombotic agents have been known to be significantly associated with delayed post-ESD bleeding. Therefore, in this study, the reason why almost all the post-ESD bleeding occurred in late phase is that we enrolled patients who were regularly taking antithrombotic agents, a risk factor for delayed bleeding. In addition, we performed meticulous hemostasis, meaning the preventive coagulation of all visible vessels, so we could reduce the rate of early post-ESD bleeding.

Although further large-scale studies are needed, according to a previous study [19] and the present study, there is a possibility that PHP can prevent early-phase bleeding in patients undergoing ESD with high risk factors. Considering that post-ESD bleeding did not occur in the PHP group in patients who stopped antithrombotic agents and PHP acted on the early phase, the efficacy of PHP might be maximized in patients who were high risk because of a large resected lesion.

In this study, diabetes mellitus, coronary artery disease, antithrombotic use, multiple antithrombotic use, and continued antithrombotic use were significant risk factors for post-ESD bleeding. In multivariate analysis, only continued antithrombotic use was an independent risk factor for post-ESD bleeding. A large resected lesion is also known to be a strong risk factor for post-ESD bleeding $[12,14]$. However, in our study, the mean resected lesion size was $48.9 \mathrm{~mm}$ in the PHP group and $46.9 \mathrm{~mm}$ in the control group, and the largest lesion was $90 \mathrm{~mm}$. In other words, the resected lesion sizes in this study were larger than average. This is the reason why only continued antithrombotic use was the most significant factor for post-ESD bleeding.

Furthermore, all cases of post-ESD bleeding in the PHP group occurred in patients with continued antithrombotic use, whereas most cases of post-ESD bleeding in the control group occurred in patients who stopped taking their antithrombotic agent. In subgroup analysis of the patients who stopped antithrombotic use, post-ESD bleeding did not occur in the PHP group ( $0 \%$ vs. $6.3 \% ; P=0.058$ ). Although there was no statistical significance, this result showed a possible role for preventing post-ESD bleeding in high risk patients with a large tumor who have stopped antithrombotic therapy. 
- Table 3 Risk factors for post-endoscopic submucosal dissection (ESD) bleeding.

\begin{tabular}{|c|c|c|c|c|c|}
\hline \multirow[t]{2}{*}{ Variables } & \multicolumn{3}{|c|}{ Univariate analysis } & \multicolumn{2}{|l|}{ Multivariate analysis } \\
\hline & $\begin{array}{l}\text { Post-ESD } \\
\text { bleeding } \\
(\mathrm{N}=9)\end{array}$ & $\begin{array}{l}\text { No bleeding } \\
(N=134)\end{array}$ & $P$ value & Odds ratio $(95 \% \mathrm{Cl})$ & $P$ value \\
\hline \multicolumn{6}{|l|}{ Patients } \\
\hline Age, mean (SD), years & $71.1(5.3)$ & $65.0(9.3)$ & 0.05 & $1.02(0.91-1.14)$ & 0.74 \\
\hline \multicolumn{3}{|l|}{ Sex, n (\%) } & \multirow[t]{3}{*}{0.45} & & \\
\hline - Male & $8(88.9)$ & $97(72.4)$ & & & \\
\hline - Female & $1(11.1)$ & $37(27.6)$ & & & \\
\hline \multicolumn{6}{|l|}{ Co-morbidity, n (\%) } \\
\hline - Hypertension & $6(66.7)$ & $60(44.8)$ & 0.30 & & \\
\hline - Diabetes mellitus & $6(66.7)$ & $28(20.9)$ & 0.01 & $2.87(0.44-18.84)$ & 0.27 \\
\hline - Coronary artery disease & $3(33.3)$ & $10(7.5)$ & 0.04 & $0.72(0.07-7.27)$ & 0.78 \\
\hline - Cerebrovascular disease & $2(22.2)$ & $12(9.0)$ & 0.22 & & \\
\hline - Chronic kidney disease & $2(22.2)$ & $5(3.7)$ & 0.06 & & \\
\hline Antithrombotic agents use, $\mathrm{n}(\%)$ & $6(66.7)$ & $32(23.9)$ & 0.01 & & \\
\hline - Aspirin & $5(55.6)$ & $17(12.7)$ & 0.01 & & \\
\hline - Clopidogrel & $3(33.3)$ & $11(8.2)$ & 0.04 & & \\
\hline - Other antiplatelets & $0(0.0)$ & $5(3.7)$ & $>0.99$ & & \\
\hline Multiple antithrombotic use, n (\%) & $2(22.2)$ & $2(1.5)$ & 0.02 & $10.26(0.60-174.93)$ & 0.11 \\
\hline \multicolumn{6}{|l|}{ Antithrombotic management, $\mathrm{n}(\%)$} \\
\hline - Continued use & $5(55.6)$ & $9(6.7)$ & $<0.001$ & $8.73(1.05-72.39)$ & 0.045 \\
\hline PHP use & $4(44.4)$ & $69(51.5)$ & 0.74 & $1.89(0.36-9.87)$ & 0.45 \\
\hline \multicolumn{6}{|l|}{ ESD procedure } \\
\hline \multicolumn{3}{|l|}{ Tumor location, $\mathrm{n}(\%)$} & \multirow[t]{4}{*}{0.58} & & \\
\hline - Lower third & $8(88.9)$ & $115(85.8)$ & & & \\
\hline - Middle third & $1(11.1)$ & $8(6.0)$ & & & \\
\hline - Upper third & $0(0.0)$ & $11(8.2)$ & & & \\
\hline Resection size, mean (SD), mm & $49.6(15.8)$ & $47.8(10.0)$ & 0.62 & & \\
\hline Resection time, mean (SD), minutes & $31.2(27.2)$ & $28.1(29.1)$ & 0.76 & & \\
\hline Preventive coagulation time, mean (SD), minutes & $6.2(2.5)$ & $6.0(5.4)$ & 0.90 & & \\
\hline En bloc resection, $\mathrm{n}(\%)$ & $9(100.0)$ & $133(99.3)$ & $>0.99$ & & \\
\hline Complete resection, n (\%) & $9(100.0)$ & $126(94.0)$ & $>0.99$ & & \\
\hline Curative resection, $\mathrm{n}(\%)$ & $9(100.0)$ & $121(90.3)$ & $>0.99$ & & \\
\hline Intraprocedural perforation, n (\%) & $0(0.0)$ & $0(0.0)$ & - & & \\
\hline \multicolumn{6}{|l|}{ Pathologic diagnosis } \\
\hline Tumor size, mean (SD), mm & $22.0(18.6)$ & $18.6(10.7)$ & 0.60 & & \\
\hline \multicolumn{3}{|l|}{ Histology, n (\%) } & \multirow[t]{3}{*}{0.72} & & \\
\hline - Adenoma & $3(33.3)$ & $39(29.1)$ & & & \\
\hline - Carcinoma & $6(66.7)$ & $95(70.9)$ & & & \\
\hline
\end{tabular}


In previous studies, continued use of antithrombotic agents posed a significantly higher bleeding risk than their withdrawal $[12,13,28]$. Moreover, the post-ESD bleeding rate reached $57.3 \%$ in patients who continued using antithrombotic agents $[10,11,29]$, and the odds ratio of bleeding with continuation of antithrombotic agents was up to 8.39 in a meta-analysis [8]. The bleeding risk might therefore be different in patients with continued or interrupted antithrombotic use. As a result, we hypothesized that using PHP might help prevent post-ESD bleeding in high risk patients who discontinue antithrombotic use.

Several efforts have been made to prevent post-ESD bleeding $[16,17,30-32]$. Preventive coagulation of visible vessels on iatrogenic ulcers and proton pump inhibitor (PPI) administration after ESD are generally recommended to reduce post-ESD bleeding [31,32]. Previous studies have shown that closure of large mucosal defects after ESD with an overstitch endoscopic suturing device decreased the need for hospitalization [17]. Additionally, routine mucosal closure with a detachable snare and clips after ESD promotes earlier healing of iatrogenic ulcers [16]. However, this method could not significantly decrease post-ESD bleeding. Furthermore, these procedures require a lot of time and high skill. PHP is easier to use because it is simply sprayed onto the surface of the post-ESD ulcer site using a catheter. Moreover, it does not cause additional secondary tissue injury owing to its non-contact application. In our experience, the time for spraying PHP is short and the method of PHP application is easy. Therefore, beginners in ESD can easily apply PHP to prevent post-ESD bleeding of iatrogenic ulcers. PHP application is still a good candidate for preventing earlyphase bleeding after ESD in high risk patients. In particular, $\mathrm{PHP}$ is useful to endoscopists with less experience in ESD.

This study had some limitations. First, the participating institutions were all high volume referral centers. Therefore, all participating endoscopists were skilled experts in gastric ESD. The participating endoscopists had previous experience of over 500 gastric ESD procedures. We believe that this is one of the reasons for the extremely low post-ESD bleeding rate, even in the high risk patients in this study. Therefore, the efficacy of PHP will be maximized in countries where ESD is not yet popular and endoscopists are still in the early phase of the ESD learning curve.

Second, this was an open-label RCT, and complete exclusion of some potential biases was impossible. In addition, the endoscopist might have performed more meticulous hemostasis than usual. In addition, the expectations of sample size according to our previous data on post-ESD bleeding rate might have been inaccurate because of the long period of collection and possible confounders. Therefore, a multicenter study of a much larger sample size will be required to determine if there is a meaningful difference in the preventive efficacy of PHP on post-ESD bleeding in high risk patients. Moreover, the sample size was not sufficient for multivariate analysis because post-ESD bleeding occurred in only nine patients, which is a small number to evaluate the risk factors post-ESD bleeding.

Nevertheless, this is the first multicenter RCT to evaluate the efficacy of PHP in preventing post-ESD bleeding. In particular, this study was meaningful in terms of targeting high risk patients who were taking antithrombotic agents and with large resected lesions. In addition, we found that there was a tendency toward decreased early-phase post-ESD bleeding rate in the PHP group in high risk patients with large lesions who discontinued antithrombotic use.

In conclusion, the present study could not demonstrate a significant effect of PHP on the prevention of post-ESD bleeding in high risk patients.

\section{Clinical trial}

Trial Registration: ClinicalTrials.gov | Registration number (trial ID): NCT03169569 | Type of study: Prospective, Randomized, Multi-Center Study

\section{Competing interests}

The authors declare that they have no conflict of interest.

\section{References}

[1] Gu L, Khadaroo PA, Chen L et al. Comparison of long-term outcomes of endoscopic submucosal dissection and surgery for early gastric cancer: a systematic review and meta-analysis. J Gastrointest Surg 2019; 23: 1493-1501

[2] Li H, Feng LQ, Bian YY et al. Comparison of endoscopic submucosal dissection with surgical gastrectomy for early gastric cancer: An updated meta-analysis. World J Gastrointest Oncol 2019; 11: 161-171

[3] Kim Y], Park DK. Management of complications following endoscopic submucosal dissection for gastric cancer. World J Gastrointest Endosc 2011; 3: 67-70

[4] Goto O, Fujishiro M, Oda I et al. A multicenter survey of the management after gastric endoscopic submucosal dissection related to postoperative bleeding. Dig Dis Sci 2012; 57: 435-439

[5] Koh R, Hirasawa K, Yahara S et al. Antithrombotic drugs are risk factors for delayed postoperative bleeding after endoscopic submucosal dissection for gastric neoplasms. Gastrointest Endosc 2013; 78: 476483

[6] Yano T, Tanabe S, Ishido K et al. Different clinical characteristics associated with acute bleeding and delayed bleeding after endoscopic submucosal dissection in patients with early gastric cancer. Surg Endosc 2017: doi:10.1007/s00464-017-5513-1

[7] Park YM, Cho E, Kang HY et al. The effectiveness and safety of endoscopic submucosal dissection compared with endoscopic mucosal resection for early gastric cancer: a systematic review and metaanalysis. Surg Endosc 2011; 25: 2666-2677

[8] Dong J, Wei K, Deng J et al. Effects of antithrombotic therapy on bleeding after endoscopic submucosal dissection. Gastrointest Endosc 2017; 86: 807-816

[9] Shindo Y, Matsumoto S, Miyatani H et al. Risk factors for postoperative bleeding after gastric endoscopic submucosal dissection in patients under antithrombotics. World J Gastrointest Endosc 2016; 8: 349-356

[10] Takeuchi T, Ota K, Harada S et al. The postoperative bleeding rate and its risk factors in patients on antithrombotic therapy who undergo gastric endoscopic submucosal dissection. BMC Gastroenterol 2013; 13: 136 
[11] Ono S, Fujishiro $\mathrm{M}$, Yoshida $\mathrm{N}$ et al. Thienopyridine derivatives as risk factors for bleeding following high risk endoscopic treatments: Safe Treatment on Antiplatelets (STRAP) study. Endoscopy 2015; 47: 632637

[12] Libanio D, Costa MN, Pimentel-Nunes P et al. Risk factors for bleeding after gastric endoscopic submucosal dissection: a systematic review and meta-analysis. Gastrointest Endosc 2016; 84: 572-586

[13] Sato C, Hirasawa K, Koh R et al. Postoperative bleeding in patients on antithrombotic therapy after gastric endoscopic submucosal dissection. World J Gastroenterol 2017; 23: 5557-5566

[14] Kataoka Y, Tsuji Y, Sakaguchi Y et al. Bleeding after endoscopic submucosal dissection: Risk factors and preventive methods. World ] Gastroenterol 2016; 22: 5927-5935

[15] Hasuike N, Ono H, Boku N et al. A non-randomized confirmatory trial of an expanded indication for endoscopic submucosal dissection for intestinal-type gastric cancer (cT1a): the Japan Clinical Oncology Group study (JCOG0607). Gastric Cancer 2018; 21: 114-123

[16] Lee BI, Kim BW, Kim HK et al. Routine mucosal closure with a detachable snare and clips after endoscopic submucosal dissection for gastric epithelial neoplasms: a randomized controlled trial. Gut Liver 2011; 5: 454-459

[17] Kantsevoy SV, Bitner M, Mitrakov AA et al. Endoscopic suturing closure of large mucosal defects after endoscopic submucosal dissection is technically feasible, fast, and eliminates the need for hospitalization (with videos). Gastrointest Endosc 2014; 79: 503-507

[18] Tsuji Y, Fujishiro M, Kodashima S et al. Polyglycolic acid sheets and fibrin glue decrease the risk of bleeding after endoscopic submucosal dissection of gastric neoplasms (with video). Gastrointest Endosc 2015; 81: 906-912

[19] Hahn KY, Park JC, Lee YK et al. Efficacy of hemostatic powder in preventing bleeding after gastric endoscopic submucosal dissection in high-risk patients. J Gastroenterol Hepatol 2018; 33: 656-663

[20] Choe YH, Park JC, Kim Y] et al. Sa1227. Predictive risk model for postendoscopic submucosal dissection ulcer bleeding of stomach: single center cohort study. Gastrointest Endosc 2018; 87: AB173-AB174

[21] Prei JC, Barmeyer C, Burgel N et al. EndoClot polysaccharide hemostatic system in nonvariceal gastrointestinal bleeding: results of a prospective multicenter observational pilot study. J Clin Gastroenterol 2016; 50: e95-e100
[22] Chung IK, Lee JH, Lee SH et al. Therapeutic outcomes in 1000 cases of endoscopic submucosal dissection for early gastric neoplasms: Korean ESD Study Group multicenter study. Gastrointest Endosc 2009; 69: $1228-1235$

[23] Takizawa K, Oda I, Gotoda T et al. Routine coagulation of visible vessels may prevent delayed bleeding after endoscopic submucosal dissection-an analysis of risk factors. Endoscopy 2008; 40: 179-183

[24] Beg S, Al-Bakir I, Bhuva M et al. Early clinical experience of the safety and efficacy of EndoClot in the management of non-variceal upper gastrointestinal bleeding. Endosc Int Open 2015; 3: E605-E609

[25] Kaehler G, Dutenhoefner C, Magdeburg R. Endoscopic application of polysaccharide powder for hemostasis in anticoagulated pigs (with video). Gastrointest Endosc 2015; 82: 161-163

[26] Yano T, Tanabe S, Ishido K et al. Different clinical characteristics associated with acute bleeding and delayed bleeding after endoscopic submucosal dissection in patients with early gastric cancer. Surg Endosc 2017; 31: 4542-4550

[27] Nam HS, Choi CW, Kim SJ et al. Risk factors for delayed bleeding by onset time after endoscopic submucosal dissection for gastric neoplasm. Sci Rep 2019; 9: 2674

[28] Kono Y, Obayashi Y, Baba Y et al. Postoperative bleeding risk after gastric endoscopic submucosal dissection during antithrombotic drug therapy. J Gastroenterol Hepatol 2018; 33: 453-460

[29] Shindo Y, Matsumoto S, Miyatani H et al. Risk factors for postoperative bleeding after gastric endoscopic submucosal dissection in patients under antithrombotics. World J Gastrointest Endosc 2016; 8: 349-356

[30] Kataoka Y, Tsuji Y, Hirasawa K et al. Endoscopic tissue shielding to prevent bleeding after endoscopic submucosal dissection: a prospective multicenter randomized controlled trial. Endoscopy 2019; 51: 619-627

[31] Takizawa K, Oda I, Gotoda T et al. Routine coagulation of visible vessels may prevent delayed bleeding after endoscopic submucosal dissection. An analysis of risk factors. Endoscopy 2008; 40: 179-183

[32] Uedo N, Takeuchi Y, Yamada T et al. Effect of a proton pump inhibitor or an $\mathrm{H} 2$-receptor antagonist on prevention of bleeding from ulcer after endoscopic submucosal dissection of early gastric cancer: a prospective randomized controlled trial. Am J Gastroenterol 2007; 102: $1610-1616$ 